\title{
Automorphic forms of higher order
}

\author{
Anton Deitmar \& Nikolaos Diamantis
}

\begin{abstract}
In this paper a theory of Hecke operators for higher order modular forms is established. The definition of higher order forms is extended beyond the realm of parabolic invariants. A canonical inner product is introduced. The role of representation theoretic methods is clarified and, motivated by higher order forms, new convolution products of L-functions are introduced.
\end{abstract}

\section{Contents}

1 Higher invariants and Hecke operators 3

1.1 Higher invariants . . . . . . . . . . . . . . . 3

1.2 Hecke operators . . . . . . . . . . . . . . . 4

1.3 Unitary Hecke modules . . . . . . . . . . . . . . . . . 9

1.4 Lowering the order . . . . . . . . . . . . . . . . . 12

2 Automorphic forms $\quad 14$

2.1 Modular forms . . . . . . . . . . . . . . . . . 14

2.2 Cusp forms . . . . . . . . . . . . . . 17

2.3 Fourier-Taylor expansion . . . . . . . . . . . . . . . . . 18

2.4 Intervention of Lie groups . . . . . . . . . . . . . . . . 19

2.5 No intervention of adèles . . . . . . . . . . . . . . . 21

3 L-functions $\quad 22$ 
3.1 Higher order cusp forms . . . . . . . . . . . . . . . 22

3.2 Convolution of L-functions . . . . . . . . . . . . . . . . . 23

\section{Introduction}

Higher-order modular forms have in recent years arisen in various contexts and they have been studied as analytic functions $[9,12]$. In particular their spaces $[1,5,6,7]$ have been investigated. Parallel to that, L-functions were attached to higher-order forms $[2,4,8,10]$ and some of their basic aspects studied. In [3], a cohomology theory for higher invariants is developed, and an Eichler-Shimura isomorphism for higher order forms is established.

The current paper serves the following purposes,

- to establish a theory of Hecke operators on higher order forms,

- to extend the definition of higher forms beyond parabolic invariants,

- to clarify the role of representation theoretic methods in the theory, and

- to introduce new convolution products of L-functions of higher forms.

The first item fills a long-standing gap in the theory of higher order forms by constructing a natural Hecke action. This is surprising, as there is no adelic counterpart of higher order forms. The Hecke operators form bounded self-adjoint operators on direct limits of spaces of higher order forms. It is an on-going long-term project of the authors to better understand the spectral decompositions of Hecke operators. For the second item, one gets that in the case of higher order forms, the crucial Fourier expansion is replaced by a "Fourier-Taylor-expansion" which is introduced in this paper. For the third item, representation theoretic methods, it turns out that a intervention of Lie groups, as Dieudonné terms it, is possible in the theory and, in fact, higherorder forms can be incorporated into the same representational context as the classical automorphic forms. One would thus expect a distinction from classical forms in the intervention of adeles. Indeed, it turns out that there is no intervention of adeles, as there are no higher forms on the adelic level. The 
last item in the list, the convolution product, is inspired by the second insofar as the L-functions of higher-order forms are special cases of the convolution products. We show analyticity and functional equation in greater generality.

\section{Higher invariants and Hecke operators}

\section{$1.1 \quad$ Higher invariants}

Let $R$ be a commutative ring with unit and let $\Gamma$ be a group. Let $R[\Gamma]$ be the group algebra and $I_{\Gamma} \subset R[\Gamma]$ the augmentation ideal. Note that $I_{\Gamma}$ is a free

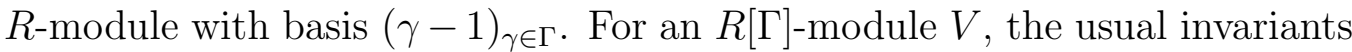
are the elements of the $R$-module $H^{0}(\Gamma, V)=V^{\Gamma}=\left\{v \in V: I_{\Gamma} v=0\right\}$. We define the set of higher invariants to be

$$
H_{q}^{0}(\Gamma, V)=\left\{v \in V: I_{\Gamma}^{q+1} v=0\right\},
$$

for $q=0,1,2, \ldots$, where $I_{\Gamma}^{k}$ is the $k$-th power of the ideal $I_{\Gamma}$. For $v \in V$ one has $v \in H_{q+1}^{0}(\Gamma, V)$ if and only if $(\gamma-1) v \in H_{q}^{0}(\Gamma, V)$ for every $\gamma \in \Gamma$. Note that if $\Gamma$ is perfect, i.e., if $[\Gamma, \Gamma]=\Gamma$, then $I_{\Gamma}^{q+1}=I_{\Gamma}$ for every $q \geq 0$ and so in that case there are no higher invariants except the usual invariants. This is due to the observation

$[a, b]-1=a b a^{-1} b^{-1}-1=(a b-b a)(b a)^{-1}=[(a-1)(b-1)-(b-1)(a-1)](b a)^{-1}$,

which shows that if $\Gamma$ is perfect, then $I_{\Gamma}^{2}=I_{\Gamma}$ and hence inductively, $I_{\Gamma}^{q+1}=$ $I_{\Gamma}$.

Proposition 1.1.1 Assume $R=\mathbb{C}$, that $\Gamma$ is a compact group, and that $V$ is a Hilbert space on which $\Gamma$ acts by a continuous representation. Then there are no higher invariants except the classical invariants, i.e., one has $H_{q}^{0}(\Gamma, V)=H^{0}(\Gamma, V)$ for every $q \geq 0$.

Proof: Let $q \geq 1$. Formally set $H_{-1}^{0}(\Gamma, V)=0$. For every $v \in H_{q}^{0}(\Gamma, V)$ the map $\gamma \mapsto(\gamma-1) v \in H_{q-1}^{0}(\Gamma, V) / H_{q-2}^{0}(\Gamma, V)$ is a group homomorphism to the additive group, as $(\gamma \tau-1) \equiv(\gamma-1)+(\tau-1) \bmod I_{\Gamma}^{2}$. Let $\gamma \in \Gamma$. Then one has $\left(\gamma^{n}-1\right) v=n(\gamma-1) v$. Since $\Gamma$ is compact, there is a sequence $n_{k} \rightarrow \infty$ 
in $\mathbb{N}$ such that $\gamma^{n_{k}}$ converges in $\Gamma$. Hence $\left(\gamma^{n_{k}}-1\right) v=n_{k}(\gamma-1) v$ converges, too, and so $(\gamma-1) v=0$. This holds for every $\gamma \in \Gamma$ and so $(\gamma-1) v \in H_{q-2}^{0}$ which implies $v \in H_{q-1}^{0}$, so $H_{q}^{0}=H_{q-1}^{0}$ and inductively $H_{q}^{0}=H_{0}^{0}$.

\subsection{Hecke operators}

We now come to Hecke operators. For this, let $(G, \Gamma)$ be a Hecke pair, i.e., $G$ is a group and $\Gamma$ is a subgroup such that for every $g \in G$ the set $\Gamma g \Gamma / \Gamma$ is finite.

\section{Examples 1.2.1}

- A classical example is $G=\mathrm{GL}_{2}(\mathbb{Q})$ and $\Gamma=\mathrm{GL}_{2}(\mathbb{Z})$.

- Let $G$ be a topological group and let $\Gamma$ be a compact open subgroup. Then $(G, \Gamma)$ is a Hecke pair, since the compact set $\Gamma g \Gamma$ can be covered by finitely many open sets of the form $x \Gamma, x \in G$.

The Hecke algebra $\mathcal{H}=\mathcal{H}_{R}(G, \Gamma)$ is the $R$-module of all functions $f$ : $\Gamma \backslash G / \Gamma \rightarrow R$ of finite support with the convolution product

$$
f * h(x)=\sum_{y \in G / \Gamma} f(y) h\left(y^{-1} x\right) .
$$

As an $R$-module, $\mathcal{H}$ is free with basis $\left(\mathbf{1}_{\Gamma g \Gamma}\right)_{g \in G}$, where $\mathbf{1}_{A}$ is the characteristic function of the set $A$. Let $V$ be an $R[G]$-module. The Hecke algebra $\mathcal{H}$ acts naturally on $V^{\Gamma}=H^{0}(\Gamma, V)$ via

$$
f . v=\sum_{y \in G / \Gamma} f(y) y v .
$$

In particular one has

$$
\mathbf{1}_{\Gamma g \Gamma} v=\sum_{j=1}^{n} g_{j} v,
$$

where $\Gamma g \Gamma=\cup_{j=1}^{n} g_{j} \Gamma$. As $v$ is $\Gamma$-invariant, this expression does not depend on the choice of the representatives $g_{j}$. 
For $q \geq 1$ and $v \in H_{q}^{0}(\Gamma, V)$, however, the expression $\sum_{j=1}^{n} g_{j} v$ will in general depend on the choice of the representatives $\left(g_{j}\right)$. Any other set of representatives is of the form $\left(g_{j} \gamma_{j}\right)$ for some $\gamma_{j} \in \Gamma$. Note that

$$
\sum_{j} g_{j} \gamma_{j} v-\sum_{j} g_{j} v=\sum_{j} g_{j} \underbrace{\left(\gamma_{j}-1\right) v}_{\in H_{q-1}^{0}(\Gamma, V) .}
$$

The group $\Gamma$ permutes the finite set $\Gamma g \Gamma / \Gamma$ by left multiplication. Let $\Gamma(g) \subset$ $\Gamma$ be the subgroup of all elements which act trivially on $\Gamma g \Gamma / \Gamma$. Then $\Gamma(g)$ is a finite index normal subgroup of $\Gamma$. Note that

$$
\Gamma(g)=\bigcap_{\gamma \in \Gamma} \gamma\left(\Gamma \cap g \Gamma g^{-1}\right) \gamma^{-1}
$$

so $\Gamma(g)$ is the biggest normal subgroup contained in $\Gamma \cap g \Gamma g^{-1}$.

Lemma 1.2.2 (a) For $v \in H_{q}^{0}(\Gamma, V)$ the sum $\sum_{j=1}^{n} g_{j} v$ lies in $H_{q}^{0}(\Gamma(g), V)$.

(b) For $v \in H_{q}^{0}(\Gamma, V)$ and any choice of elements $\gamma_{j} \in \Gamma$ the sum $\sum_{j=1}^{n} g_{j}\left(\gamma_{j}-1\right) v$ lies in $H_{q-1}^{0}(\Gamma(g), V)$.

Proof: (a) We have to show that the sum is anihilated by

$$
\left(\sigma_{0}-1\right) \cdots\left(\sigma_{q}-1\right)
$$

for any given $\sigma_{0}, \ldots, \sigma_{q} \in \Gamma(g)$. By the definition of $\Gamma(g)$ it follows that for every $j$ and every $k$ there exist $\eta_{j}^{k} \in \Gamma$ such that $\sigma_{k} g_{j}=g_{j} \eta_{j}^{k}$. Hence,

$$
\left(\sigma_{0}-1\right) \cdots\left(\sigma_{q}-1\right) \sum_{j=1}^{n} g_{j}=\sum_{j} g_{j}\left(\eta_{j}^{0}-1\right) \cdots\left(\eta_{j}^{q}-1\right) v=0 .
$$

The proof of (b) is similar.

The lemma implies that we get a well defined Hecke operator

$$
T_{\Gamma g \Gamma}: H_{q}^{0}(\Gamma, V) / H_{q-1}^{0}(\Gamma, V) \rightarrow H_{q}^{0}(\Gamma(g), V) / H_{q-1}^{0}(\Gamma(g), V) .
$$

We need to extend this construction to finite index subgroups $\Sigma \subset \Gamma$. First note that $(G, \Sigma)$ is a Hecke pair again. We abbreviate

$$
\bar{H}_{q}(\Sigma, V)=H_{q}^{0}(\Sigma, V) / H_{q-1}^{0}(\Sigma, V) .
$$


As every finite index subgroup contains a finite index normal subgroup it suffices to assume that $\Sigma$ is normal in $\Gamma$. We define $\Sigma(g)$ to be the kernel of the homomorphism $\Sigma \rightarrow \operatorname{Per}(\Gamma g \Gamma / \Sigma)$. Note that $\Gamma$ is present in the definition, although not in the notation. Then, $\Sigma(g)=\bigcap_{\gamma \in \Gamma} \gamma\left(\Sigma \cap g \Sigma g^{-1}\right) \gamma^{-1}$, and $\Sigma(g)$ is normal of finite index in $\Gamma$. We define

$$
T_{\Gamma g \Gamma}: \bar{H}_{q}(\Sigma, V) \rightarrow \bar{H}_{q}(\Sigma(g), V)
$$

as follows. Write $\Gamma g \Gamma$ as a disjoint union of $\Sigma$-cosets $\bullet_{j} h_{j} \Sigma$ and set

$$
T_{\Gamma g \Gamma} v=\frac{1}{[\Gamma: \Sigma]} \sum_{j} h_{j} v .
$$

The same reasoning as before shows the well-definedness of $T_{\Gamma g \Gamma}$. The factor $\frac{1}{[\Gamma: \Sigma]}$ will make the Hecke operator compatible with change of groups as follows. Assume $\Sigma^{\prime} \subset \Sigma$ is another finite index normal subgroup. Then $\Sigma^{\prime}(g) \subset \Sigma(g)$ as well and the inclusions

$$
\begin{array}{ccc}
H_{q}^{0}(\Sigma, V) & \subset & H_{q}^{0}\left(\Sigma^{\prime}, V\right) \\
\cup & & \cup \\
H_{q-1}^{0}(\Sigma, V) & \subset & H_{q-1}^{0}\left(\Sigma^{\prime}, V\right)
\end{array}
$$

show that there is a natural restriction homomorphism $\operatorname{res}_{\Sigma^{\prime}}^{\Sigma}: \bar{H}_{q}(\Sigma, V) \rightarrow$ $\bar{H}_{q}\left(\Sigma^{\prime}, V\right)$. As the intersection of two finite index subgroups is a finite index subgroup, these spaces form a direct system indexed by the set of all finite index normal subgroups $\Sigma$ of $\Gamma$.

Lemma 1.2.3 For any two finite index normal subgroups $\Sigma^{\prime} \subset \Sigma$ of $\Gamma$ and $g \in G$ the diagram

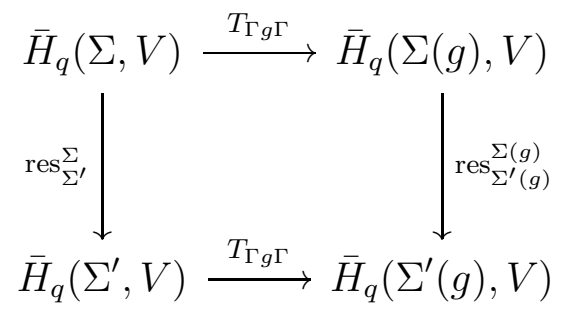

commutes. 
Proof: Note first that, as $\Sigma$ is normal in $\Gamma$, the group $\Gamma$ acts on the finite set $\Gamma g \Gamma / \Sigma$ from the right by $\gamma: h \Sigma \mapsto h \gamma \Sigma$. Therefore,

$$
|\Gamma g \Gamma / \Sigma|=\sum_{h: \Gamma g \Gamma / \Gamma}\left|\Gamma_{h \Sigma} \backslash \Gamma\right|,
$$

where $\Gamma_{h \Sigma}$ is the stabilizer of $h \Sigma$ in $\Gamma$. Note that

$$
\gamma \in \Gamma_{h \Sigma} \Leftrightarrow h \gamma \Sigma=h \Sigma \Leftrightarrow \gamma \in \Sigma,
$$

so that

$$
|\Gamma g \Gamma / \Sigma|=|\Gamma g \Gamma / \Gamma||\Sigma \backslash \Gamma| .
$$

Let $v \in H_{q}^{0}(\Sigma, V)$. Then $v$ represents the class $[v]=v+H_{q-1}^{0}(\Sigma, V)$ in $\bar{H}_{q}(\Sigma, V)$. The element $\operatorname{res}_{\Sigma^{\prime}}^{\Sigma}([v])$ is also represented by the same $v$, so that $T_{\Gamma g \Gamma} \operatorname{res}_{\Sigma^{\prime}}^{\Sigma}([v])$ is represented by

$$
\frac{1}{\left[\Gamma: \Sigma^{\prime}\right]} \sum_{h: \Gamma g \Gamma / \Sigma^{\prime}} h v .
$$

On the other hand, $T_{\Gamma g \Gamma}([v])$ is represented by

$$
\frac{1}{[\Gamma: \Sigma]} \sum_{i} k_{i} v
$$

where $\Gamma g \Gamma /=\cup_{i} k_{i} \Sigma$. Suppose that $\Sigma=\cup_{j} g_{j} \Sigma^{\prime}$. Then, by Lemma 1.2.2(b), the sum (1) equals

$$
\frac{1}{[\Gamma: \Sigma]} \sum_{i}\left(\frac{1}{\left[\Sigma: \Sigma^{\prime}\right]} \sum_{j} k_{i} g_{j} v\right)
$$

modulo $H_{q-1}^{0}(\Sigma(g), V)$. As $\left[\Gamma: \Sigma^{\prime}\right]=[\Gamma: \Sigma]\left[\Sigma: \Sigma^{\prime}\right]$ and $\Gamma g \Gamma=\cup_{i, j} k_{i} g_{j} \Sigma^{\prime}$ the last sum equals $\frac{1}{\left[\Gamma: \Sigma^{\prime}\right]} \sum_{\Gamma g \Gamma / \Sigma^{\prime}} l v$ modulo $H_{q-1}^{0}(\Sigma(g), V)$ and its restriction to $\Sigma^{\prime}$ equals the same sum modulo $H_{q-1}^{0}\left(\Sigma^{\prime}(g), V\right)$.

In applications, it will be necessary to consider subsystems like the system of congruence subgroups defined as follows. A subgroup $\Sigma$ of $\Gamma$ is called a congruence subgroup, if it contains a subgroup of the form $\Gamma\left(g_{1}\right)\left(g_{2}\right) \ldots\left(g_{n}\right)$ for some $g_{1}, \ldots g_{n} \in G$. 
Lemma 1.2.4 The intersection of two congruence subgroups is a congruence subgroup.

Proof: We claim that

$$
\Gamma\left(g_{1}\right) \ldots\left(g_{n}\right) \bigcap \Gamma\left(h_{1}\right) \ldots\left(h_{m}\right)
$$

contains

$$
\Gamma\left(g_{1}\right) \ldots\left(g_{n}\right)\left(h_{1}\right) \ldots\left(h_{m}\right) .
$$

We spell out the argument in the case $m=n=1$ and leave the obvious iteration to the reader. Recall that $\Sigma(g)$ is defined as the kernel of the map $\Sigma \rightarrow \operatorname{Per}(\Gamma g \Gamma / \Sigma)$. So a given $\gamma \in \Gamma$ lies in $\Gamma(g)$ if and only if $\gamma$ acts trivially on $\Gamma g \Gamma / \Gamma$. It lies in $\Gamma(g)(h)$ iff it also acts trivially on $\Gamma h \Gamma / \Gamma(g)$. But then it acts trivially on $\Gamma h \Gamma / \Gamma$, and hence $\gamma$ lies in $\Gamma(g) \cap \Gamma(h)$.

Let

$$
L_{q}(\Gamma, V)=\underset{\Sigma}{\lim _{\Sigma}} \bar{H}_{q}(\Sigma, V),
$$

where the limit is taken over all normal congruence subgroups $\Sigma$ of $\Gamma$. Further, let $L_{q}^{\text {all }}(\Gamma, V)$ denote the same direct limit, but now over all finite index normal subgroups of $\Gamma$. The lemma above shows that one gets a well defined operator

$$
T_{\Gamma g \Gamma}: L_{q}(\Gamma, V) \rightarrow L_{q}(\Gamma, V),
$$

and likewise for $L_{q}^{\text {all }}(\Gamma, V)$. For the rest of the section, we consider the case $L_{q}(\Gamma, V)$ only, but everything will as well apply to $L_{q}^{\text {all }}(\Gamma, V)$.

Proposition 1.2.5 The map $\mathbf{1}_{\Gamma g \Gamma} \mapsto T_{\Gamma g \Gamma}$ extends uniquely to a representation of the Hecke algebra $\mathcal{H}(G, \Gamma)$ on $L_{q}(\Gamma, V)$.

Proof: Uniqueness is clear as the $1_{\Gamma g \Gamma}$ span the Hecke algebra. We only have to show that the ensuing linear map is a representation. For this we write it in a different manner. Let $f \in \mathcal{H}(G, \Gamma)$ and let $v \in \bar{H}_{q}(\Sigma, V)$. Define

$$
f . v=\frac{1}{[\Gamma: \Sigma]} \sum_{y \in G / \Sigma} f(y) y v .
$$


Then $f . v$ lies in $\bar{H}_{q}\left(\Sigma^{\prime}, V\right)$, where $\Sigma^{\prime}=\Sigma\left(g_{1}\right) \cap \cdots \cap \Sigma\left(g_{n}\right)$ and the support of $f$ is contained in $\Gamma g_{1} \Gamma \cup \cdots \cup \Gamma g_{n} \Gamma$. This is an action of $\mathcal{H}$ which extends the above map.

Remark. A given $g \in G$ maps $H_{q}^{0}(\Sigma, V)$ to $H_{q}^{0}\left(g \Sigma g^{-1}, V\right) \subset H_{q}^{0}(\Sigma \cap$ $\left.g \Sigma g^{-1}, V\right)$ and thus it maps $\bar{H}_{q}(\Sigma, V)$ to $\bar{H}_{q}\left(\Sigma \cap g \Sigma g^{-1}, V\right)$. For $\Sigma^{\prime} \subset \Sigma$ the diagram

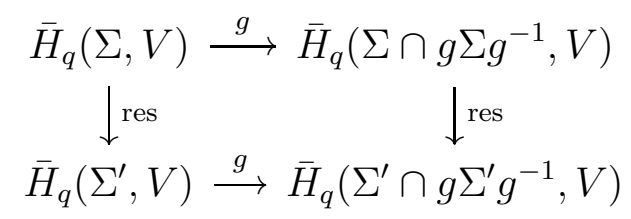

commutes. Therefore the group $G$ acts on the limit $L_{q}(\Gamma, V)$. It is sometimes easier to understand $L_{q}(\Gamma, V)$ as a $G$-module rather that a Hecke module.

\subsection{Unitary Hecke modules}

Suppose now that for every congruence subgroup $\Sigma$ the space $\bar{H}_{q}(\Sigma, V)$ is a Hilbert space in such a way that

- $\operatorname{res}_{\Sigma^{\prime}}^{\Sigma}: \bar{H}_{q}(\Sigma, V) \rightarrow \bar{H}_{q}\left(\Sigma^{\prime}, V\right)$ is an isometry if $\Sigma^{\prime} \subset \Sigma$, and

- for each $g \in G$ the map $g: \bar{H}_{q}(\Sigma, V) \rightarrow \bar{H}_{q}\left(g \Sigma g^{-1}, V\right)$, induced by the action of $g$ on $V$, is a unitary map.

The first condition gives $L_{q}(\Gamma, V)$ the structure of a pre-Hilbert space. The second implies that $G$ acts on this pre-Hilbert space by unitary maps. If this is the case for every $q \geq 1$, we call $V$ a unitary Hecke module.

Theorem 1.3.1 Let $V$ be a unitary Hecke module. For each $g \in G$ the operator $T_{\Gamma g \Gamma}$ is a bounded operator on the pre-Hilbert space $L_{q}(\Gamma, V)$. The operator norm satisfies

$$
\|T\| \leq|\Gamma g \Gamma / \Gamma| .
$$

The adjoint of $T_{\Gamma g \Gamma}$ is

$$
T_{\Gamma g \Gamma}^{*}=\frac{|\Sigma g \Sigma / \Sigma|}{\left|\Sigma g^{-1} \Sigma / \Sigma\right|} T_{\Gamma g^{-1} \Gamma} .
$$


In particular, if $\Gamma g \Gamma=\Gamma g^{-1} \Gamma$, then $T_{\Gamma g \Gamma}$ is self-adjoint.

If there exists a locally compact group $G^{*}$ with $G \subset G^{*}$ such that $\Gamma$ is a lattice in $G^{*}$, then we have

$$
T_{\Gamma g \Gamma}^{*}=T_{\Gamma g^{-1} \Gamma}
$$

for every $g \in G$.

Proof: For $v \in \bar{H}_{q}(\Sigma, V)$ with $\|v\|=1$ one has

$$
\begin{aligned}
\left\|T_{\Gamma g \Gamma} v\right\| & =\frac{1}{[\Gamma: \Sigma]}\left\|\sum_{h: \Gamma g \Gamma / \Sigma} h v\right\| \\
& \leq \frac{1}{[\Gamma: \Sigma]} \sum_{h: \Gamma g \Gamma / \Sigma} \underbrace{\|h v\|}_{=\|v\|=1} \\
& =\frac{|\Gamma g \Gamma / \Sigma|}{[\Gamma: \Sigma]}=|\Gamma g \Gamma / \Gamma| .
\end{aligned}
$$

To compute the adjoint, let $v, w \in \bar{H}_{q}(\Sigma, V)$. Then

$$
\begin{aligned}
\left\langle T_{\Gamma g \Gamma} v, w\right\rangle & =\frac{1}{[\Gamma: \Sigma]} \sum_{h: \Gamma g \Gamma / \Sigma}\langle h v, w\rangle \\
& =\frac{1}{[\Gamma: \Sigma]} \sum_{h: \Sigma \backslash \Gamma g \Gamma / \Sigma} \sum_{\sigma: \Sigma / \Sigma_{h \Sigma}}\langle\sigma h v, w\rangle \\
& =\frac{1}{[\Gamma: \Sigma]} \sum_{h: \Sigma \backslash \Gamma g \Gamma / \Sigma} \sum_{\sigma: \Sigma / \Sigma_{h \Sigma}}\left\langle h v, \sigma^{-1} w\right\rangle,
\end{aligned}
$$

where $\Sigma_{h \Sigma}$ is the stabilizer in $\Sigma$ of the coset $h \Sigma$ in the set $G / \Sigma$. We have $\sigma^{-1} w \equiv w$ modulo $H_{q-1}^{0}$, so the last line equals

$$
\frac{1}{[\Gamma: \Sigma]} \sum_{h: \Sigma \backslash \Gamma g \Gamma / \Sigma}|\Sigma h \Sigma / \Sigma|\langle h v, w\rangle,
$$

where we have used $|\Sigma h \Sigma / \Sigma|=\left|\Sigma / \Sigma_{h \Sigma}\right|$. Let $h=\gamma_{1} g \gamma_{2}$ with $\gamma_{1}, \gamma_{2} \in \Gamma$. Then

$$
\begin{aligned}
|\Sigma h \Sigma / \Sigma| & =\left|\Sigma \gamma_{1} g \gamma_{2} \Sigma / \Sigma\right| \\
& =\left|\gamma_{1} \Sigma g \gamma_{2} \Sigma / \Sigma\right| \\
& =\left|\Sigma g \gamma_{2} \Sigma / \Sigma\right|
\end{aligned}
$$


as the map $x \Sigma \mapsto \gamma_{1} x \Sigma$ is a bijection on $G / \Sigma$. Further, as $\Sigma$ is normal in $\Gamma$, the group $\Gamma$ acts on $G / \Sigma$ via $x \Sigma \mapsto x \gamma \Sigma$. Hence we get

$$
|\Sigma h \Sigma / \Sigma|=|\Sigma g \Sigma / \Sigma|
$$

And so

$$
\left\langle T_{\Gamma g \Gamma} v, w\right\rangle=\frac{|\Sigma g \Sigma / \Sigma|}{[\Gamma: \Sigma]} \sum_{h: \Sigma \backslash \Gamma g \Gamma / \Sigma}\left\langle v, h^{-1} w\right\rangle .
$$

If $h$ runs through a set of representatives of $\Sigma \backslash \Gamma g \Gamma / \Sigma$, then $h^{-1}$ runs through a set of representatives of $\Sigma \backslash \Gamma g^{-1} \Gamma / \Sigma$. It follows

$$
\left\langle T_{\Gamma g \Gamma} v, w\right\rangle=\frac{|\Sigma g \Sigma / \Sigma|}{[\Gamma: \Sigma]} \sum_{h: \Sigma \mid \Gamma g^{-1} \Gamma / \Sigma}\langle v, h w\rangle .
$$

Repeating the same argument with $g^{-1}$ in place of $g$ yields

$$
\left\langle T_{\Gamma g \Gamma} v, w\right\rangle=\frac{|\Sigma g \Sigma / \Sigma|}{\left|\Sigma g^{-1} \Sigma / \Sigma\right|}\left\langle v, T_{\Gamma g^{-1} \Gamma} w\right\rangle,
$$

or

$$
T_{\Gamma g \Gamma}^{*}=\frac{|\Sigma g \Sigma / \Sigma|}{\left|\Sigma g^{-1} \Sigma / \Sigma\right|} T_{\Gamma g^{-1} \Gamma}
$$

as claimed.

Suppose $\Gamma g \Gamma=\Gamma g^{-1} \Gamma$ and let $c=\frac{|\Sigma g \Sigma / \Sigma|}{\left|\Sigma g^{-1} \Sigma / \Sigma\right|}$. We have to show that $c=1$. With $T=T_{\Gamma g \Gamma}$ we have $T^{*}=c T$, so $T=\left(T^{*}\right)^{*}=c T^{*}=c^{2} T$. As $c>0$ we conclude $c=1$.

Finally, assume the existence of $G^{*}$. Note that $\Sigma$ is a lattice in $G^{*}$ as well and that

$$
|\Sigma g \Sigma / \Sigma|=\left|\Sigma / \Sigma \cap g \Sigma g^{-1}\right|
$$

as the set $\Sigma g \Sigma / \Sigma$ is one orbit under the left translation action of $\Sigma$ and the stabilizer of the point $g \Sigma / \Sigma$ is $\Sigma \cap g \Sigma g^{-1}$. Accordingly,

$$
\left|\Sigma g^{-1} \Sigma / \Sigma\right|=\left|\Sigma / \Sigma \cap g^{-1} \Sigma g\right|=\left|g \Sigma g^{-1} / \Sigma \cap g \Sigma g^{-1}\right| .
$$

Let $\mu$ be the left Haar measure on $G^{*}$, then $\mu\left(g \Sigma g^{-1} \backslash G^{*}\right)=\mu\left(\Sigma \backslash G^{*}\right)$ and so

$$
\begin{array}{r}
\mu\left(G^{*} /\left(\Sigma \cap g \Sigma g^{-1}\right)\right)\left|g \Sigma g^{-1} / \Sigma \cap g \Sigma g^{-1}\right|=\mu\left(G^{*} / g \Sigma g^{-1}\right)=\mu\left(\Sigma \backslash G^{*}\right) \\
=\mu\left(G^{*} /\left(\Sigma \cap g \Sigma g^{-1}\right)\right)\left|\Sigma / \Sigma \cap g \Sigma g^{-1}\right|
\end{array}
$$


This implies the claim.

A Hecke pair $(G, \Gamma)$ is called unimodular, if

$$
|\Gamma g \Gamma / \Gamma|=|\Gamma \backslash \Gamma g \Gamma|
$$

holds for every $g \in G$.

Corollary 1.3.2 Let $(G, \Gamma)$ be a Hecke pair such that there exists a locally compact group $G^{*}$ as in the theorem, then the pair $(G, \Gamma)$ is unimodular.

Proof: The last part of the proof of the theorem gives $|\Gamma g \Gamma / \Gamma|=\left|\Gamma g^{-1} \Gamma / \Gamma\right|$. The inversion $h \mapsto h^{-1}$ induces a bijection between $\Gamma g^{-1} \Gamma / \Gamma$ and $\Gamma \backslash \Gamma g \Gamma$.

Note that the condition

$$
\Gamma g \Gamma=\Gamma g^{-1} \Gamma
$$

is satisfied in important examples like the Hecke pairs $\left(\mathrm{SL}_{2}(\mathbb{Q}), \mathrm{SL}_{2}(\mathbb{Z})\right)$ or $\left(\mathrm{PGL}_{2}(\mathbb{Q}), \mathrm{PGL}_{2}(\mathbb{Z})\right)$. Note further, that this condition implies that the Hecke algebra $\mathcal{H}(G, \Gamma)$ is commutative, i.e., that $(G, \Gamma)$ is a Gelfand pair.

Example 1.3.3 For the sake of completeness we give an example of a Hecke pair $(G, \Gamma)$ which is not unimodular. Let $p$ be a prime and let $G$ be the semidirect product $\mathbb{Q}_{p} \rtimes \mathbb{Q}_{p}^{\times}$. So $G$ is the topological space $\mathbb{Q}_{p} \times \mathbb{Q}_{p}^{\times}$with the multiplication $(x, y)\left(x^{\prime}, y^{\prime}\right)=\left(x+y x^{\prime}, y y^{\prime}\right)$. Let $\Gamma$ be the compact open subgroup $\mathbb{Z}_{p} \rtimes \mathbb{Z}_{p}^{\times}$and let $g=(0, p)$. Then $|\Gamma g \Gamma / \Gamma|=p$, whereas $|\Gamma \backslash \Gamma g \Gamma|=1$.

Remark. Note that if $V$ is a unitary Hecke module, then the representation of $G$ on the pre-Hilbert space $L_{q}(\Gamma, V)$ is unitary.

\subsection{Lowering the order}

There is a canonical injective linear map

$$
l_{q}=l_{V, \Sigma, q}: \bar{H}_{q}(\Sigma, V) \hookrightarrow \operatorname{Hom}(\Gamma, \mathbb{C}) \otimes \bar{H}_{q-1}(\Sigma, V)
$$


given as follows: first note that there is a canonical isomorphism $\operatorname{Hom}(\Gamma, \mathbb{C}) \otimes$ $\bar{H}_{q-1}(\Sigma, V) \stackrel{\cong}{\longrightarrow} \operatorname{Hom}\left(\Gamma, \bar{H}_{q-1}(\Sigma, V)\right)$. Using this, we can define $l_{q}$ as

$$
l_{q}(v)(\gamma)=(\gamma-1) v .
$$

This indeed is well defined as for $\gamma, \tau \in \Sigma$ one has $(\gamma \tau-1) \equiv(\gamma-1)+(\tau-1)$ $\bmod I_{\Sigma}^{2}$. We call $l_{q}$ the order lowering homomorphism. It can be used to establish a unitary structure as is shown in the next section.

In the following sections we will give examples for spaces $V$ to which the Hecke calculus applies. For each of these spaces the following problems arise.

- Determine the spectral decomposition of $T_{\Gamma g \Gamma}$ on the Hilbert completion of $L_{q}(\Gamma, V)$. If the Hecke algebra is commutative, one can give a simultaneous spectral decomposition. Are the Hecke operators compact?

- Determine the difference that it makes for the spectrum of the Hecke algebra whether one starts with congruence subgroups only or all finite index normal subgroups $\Sigma$. This problem should be related to the Selberg conjecture.

- Determine the action of the Hecke algebra in terms of Fourier-Taylor expansions (defined below) and on the ensuing $L$-functions.

Instead of the Hecke-action, it is sometimes more useful to consider the $G$ action on $L_{q}(\Gamma, V)$. Note that the order-lowering homomorphism can be iterated to

$$
\bar{H}_{q}(\Sigma, V) \hookrightarrow \operatorname{Hom}(\Sigma, \mathbb{C})^{\otimes q} \otimes H^{0}(\Sigma, V) .
$$

Let

$$
H_{\Gamma} \stackrel{\text { def }}{=} \lim _{\vec{\Sigma}} \operatorname{Hom}(\Sigma, \mathbb{C}) .
$$

Then $H_{\Gamma}$ is a $G$-module and, taking limits, one gets an injection of $G$ modules,

$$
L_{q}(\Gamma, V) \hookrightarrow\left(H_{\Gamma}\right)^{\otimes q} \otimes V^{\infty},
$$

where $V^{\infty}=\bigcup_{\Sigma} V^{\Sigma}$. Sometimes this map will be surjective on the Hilbert space completions. Thus the spectral problem decomposes into 
- determining the spectral decomposition of the module $H_{\Gamma}$, resp. its Hilbert space completion, and

- determining the decomposition of tensor products.

These aims will be pursued in future work.

\section{Automorphic forms}

\section{$2.1 \quad$ Modular forms}

For any ring $R$, let $G_{R}=\mathrm{SL}_{2}(R)$. The Lie group $G_{\mathbb{R}}$ acts by linear fractionals on the upper half plane $\mathbb{H}$ in $\mathbb{C}$. Let $\Gamma \subset G_{\mathbb{R}}$ be a lattice. A cusp of $\Gamma$ is a parabolic fixed point in the boundary $\partial \mathbb{H}=\hat{\mathbb{R}}=\mathbb{R} \cup\{\infty\}$. For a cusp $c$, let $\Gamma_{c}$ be its stabilizer in $\Gamma$. For every cusp $c$ there exists an element $\sigma_{c}^{\Gamma} \in G_{\mathbb{R}}$ such that $\sigma_{c}^{\Gamma} \infty=c$ and the action of $\left(\sigma_{c}^{\Gamma}\right)^{-1} \Gamma_{c} \sigma_{c}^{\Gamma}$ on $\mathbb{H}$ is generated by the translation $z \mapsto z+1$. For $k \in 2 \mathbb{Z}$ and $f: \mathbb{H} \rightarrow \mathbb{C}$ define

$$
\left(\left.f\right|_{k} \gamma\right)(z)=(c z+d)^{-k} f(\gamma z)
$$

where $\gamma=\left(\begin{array}{ll}* & * \\ c & d\end{array}\right) \in G_{\mathbb{R}}$. Since we rather deal with left actions of the groups and we keep $k$ fixed, we also write $\gamma f=\left.f\right|_{k} \gamma^{-1}$.

Let $\Gamma=\mathrm{SL}_{2}(\mathbb{Z})$, then $\left(G_{\mathbb{Q}}, \Gamma\right)$ is a Gelfand pair. Let $V=\mathcal{M}_{k}$ be the complex vector space of all holomorphic functions $f: \mathbb{H} \rightarrow \mathbb{C}$ such that for every cusp $c$ of $\Gamma$ the function $\left.f\right|_{k} \sigma_{c}^{\Gamma}$ is bounded on the set $\{z \in \mathbb{H}: \operatorname{Im}(z) \geq 1\}$.

A congruence group $\Sigma \subset \Gamma$ is a subgroup which contains the group

$$
\Gamma(N)=\operatorname{ker}\left(\Gamma \rightarrow \mathrm{SL}_{n}(\mathbb{Z} / N \mathbb{Z})\right)
$$

for some $N \in \mathbb{N}$. Note that this definition coincides with our earlier definition of a congruence group for the pair $\left(G_{\mathbb{Q}}, \Gamma\right)$. Let $\Sigma \subset \Gamma$ be a congruence subgroup. The space $\mathcal{M}_{k}(\Sigma)=V^{\Sigma}=H^{0}(\Sigma, V)$ is the space of holomorphic modular forms for the group $\Sigma$. For every $f \in \mathcal{M}_{k}(\Sigma)$ and every cusp $c$ of $\Gamma$ the function $\left.z \mapsto f\right|_{k} \sigma_{c}^{\Gamma}(z)$ is periodic of period $\left|\Gamma_{c} / \Sigma_{c}\right|$. It therefore has a 
Fourier-expansion. The normalized zeroth coefficient

$$
d_{0}(f, c)=\left.\frac{1}{\left|\Gamma_{c} / \Sigma_{c}\right|} \int_{0}^{\left|\Gamma_{c} / \Sigma_{c}\right|} f\right|_{k} \sigma_{c}^{\Gamma}(z+t) d t
$$

is independent of $z \in \mathbb{H}$. Let $S_{k}(\Sigma)$ be the subspace of all cusp forms $f$ for $\Sigma$, which are characterized by the fact that for every cusp $c$ of $\Gamma$ one has

$$
d_{0}(f, c)=0 .
$$

Note that every cusp form $f$ has rapid decay at the cusps, which means that for every cusp $c$ one has $\left.f\right|_{k} \sigma_{c}^{\Gamma}(x+i y)=O\left(e^{-\alpha y}\right)$ for some $\alpha>0$.

Theorem 2.1.1 There is a canonical choice of inner products that makes $V$ a unitary Hecke module.

Proof: As a first step we have to construct a canonical inner product on $\mathcal{M}_{k}(\Sigma)$. For this note that for $f \in \mathcal{M}_{k}(\Sigma)$ and $g \in S_{k}(\Sigma)$ one has the Petersson inner product:

$$
\langle f, g\rangle_{\mathrm{Pet}}=\frac{1}{\operatorname{vol}(\Sigma \backslash \mathbb{H})} \int_{\Sigma \backslash \mathbb{H}} f(z) \overline{g(z)} y^{k} d \mu(z),
$$

where $\mu$ is the $G_{\mathbb{R}}$-invariant measure $\frac{d x d y}{y^{2}}$. We extend it to an inner product on $\mathcal{M}_{k}(\Sigma)$ as follows. The Petersson inner product defines an orthogional projection $P: \mathcal{M}_{k}(\Sigma) \rightarrow S_{k}(\Sigma)$. Let $C$ be the set of cusps of $\Gamma$. For $f, g \in \mathcal{M}_{k}(\Sigma)$ let

$$
\langle f, g\rangle_{\text {cusp }}=\frac{1}{|\Sigma \backslash C|} \sum_{c \in \Sigma \backslash C} d_{0}(f, c) \overline{d_{0}(g, c)},
$$

where the sum runs over all $\Sigma$-equivalence classes of cusps $c$. The form

$$
\langle f, g\rangle=\langle f, g\rangle_{\text {cusp }}+\langle P(f), P(g)\rangle_{\text {Pet }}
$$

is a positive definite inner product such that for $\Sigma^{\prime} \subset \Sigma$ the inclusion $\mathcal{M}_{k}(\Sigma) \hookrightarrow \mathcal{M}_{k}\left(\Sigma^{\prime}\right)$ is an isometry and for $g \in G_{\mathbb{Q}}$ the map $g: \mathcal{M}_{k}(\Sigma) \rightarrow$ $\mathcal{M}_{k}\left(g \Sigma g^{-1}\right)$ is unitary. This settles the case $q=1$. Suppose the case $q$ has been taken care of, then consider the order lowering homomorphism 
$l_{q+1}: \bar{H}_{q+1}(\Sigma, V) \hookrightarrow \operatorname{Hom}(\Sigma, \mathbb{C}) \otimes \bar{H}_{q}(\Sigma, V)$. The Eichler-Shimura map gives a canonical isomorphism

$$
\operatorname{Hom}(\Sigma, \mathbb{C}) \cong \mathcal{M}_{2}(\Sigma) \oplus \overline{S_{2}(\Sigma)}
$$

The right hand side is equipped with a canonical inner product by the above, such that for $\Sigma^{\prime} \subset \Sigma$ one has a commutative diagram

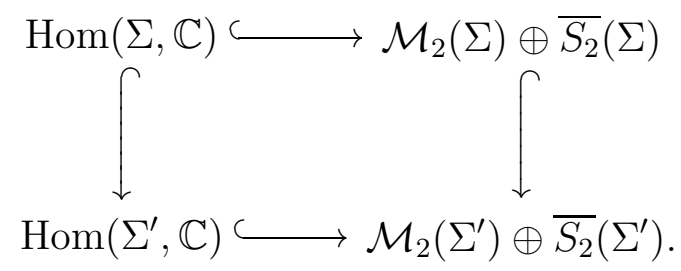

The Petersson inner product thus establishes a canonical inner product on $\operatorname{Hom}(\Sigma, \mathbb{C})$ such that the restriction $\operatorname{map} \operatorname{Hom}(\Sigma, \mathbb{C}) \rightarrow \operatorname{Hom}\left(\Sigma^{\prime}, \mathbb{C}\right)$ is isometric and for $g \in G_{\mathbb{Q}}$ the map $g: \operatorname{Hom}(\Sigma, \mathbb{C}) \rightarrow \operatorname{Hom}\left(g \Sigma g^{-1}, \mathbb{C}\right)$ is unitary. Now equip $\bar{H}_{q+1}\left(\Sigma, \mathcal{M}_{k}\right)$ with the inner product from the above injection into $\operatorname{Hom}(\Sigma, \mathbb{C}) \otimes \bar{H}_{q}(\Sigma, V)$ to make $\mathcal{M}_{k}$ a unitary Hecke module in a canonical way.

The inner product $\left\langle\cdot, \cdot>\right.$ on $\bar{H}_{q}\left(\Sigma, \mathcal{M}_{k}\right)$ can inductively be written explicitly as follows. We write $\tilde{M}_{k}^{q}=\tilde{M}_{k}^{q}(\Sigma)$ for the space $H_{q}^{0}\left(\Sigma, \mathcal{M}_{k}\right)$. Then $\bar{H}_{q}\left(\Sigma, \mathcal{M}_{k}\right)=\tilde{M}_{k}^{q} / \tilde{M}_{k}^{q-1}$. Assume that the inner product $<\cdot, \cdot>$ has been defined on $\tilde{M}_{k}^{q-1} / M_{k}^{q-2}$ and let $\left\{f_{i}\right\}_{i=1}^{d}$ be an orthonormal basis of $\tilde{M}_{k}^{q-1} / \tilde{M}_{k}^{q-2}$.

Let now $f, g \in \tilde{M}_{k}^{q} / \tilde{M}_{k}^{q-1}$ and suppose that for every $\sigma \in \Sigma$,

$$
\begin{aligned}
\left.f\right|_{k}(\sigma-1) & =\sum_{i=1}^{d} \phi_{i}(\sigma) f_{i} \\
\left.g\right|_{k}(\sigma-1) & =\sum_{i=1}^{d} \psi_{i}(\sigma) f_{i}
\end{aligned}
$$

for some $\phi_{i}, \psi_{i} \in \operatorname{Hom}(\Sigma, \mathbb{C})$. Suppose that the Eichler-Shimura isomorphism maps $\phi_{i}\left(\operatorname{resp} . \psi_{i}\right)$ to $\left(a_{i}, \bar{b}_{i}\right)$ (resp. $\left.\left(c_{i}, \bar{d}_{i}\right)\right)$ with $a_{i}, c_{i} \in \mathcal{M}_{2}$ and $b_{i}, d_{i} \in S_{2}$. Then, the definition of inner product on $\bar{H}_{q}\left(\Sigma, \mathcal{M}_{k}\right)$ can be written as

$$
\begin{aligned}
<f, g> & =\sum_{i, j}\left(<a_{i}, c_{j}><f_{i}, f_{j}>+\overline{<b_{i}, d_{j}>}<f_{i}, f_{j}>\right) \\
& =\sum_{i}\left(<a_{i}, c_{i}>+\overline{<b_{i}, d_{i}>}\right) .
\end{aligned}
$$


It should be noted that Ö. Imamoglu and C. O'Sullivan [11] have given an alternative definition of an inner product on a subspace of $\tilde{M}_{k}^{1} / \tilde{M}_{k}^{0}$ and (conjecturally) for $\tilde{M}_{k}^{q} / \tilde{M}_{k}^{q-1},(q \geq 2)$. Their construction relies on delicate analytic manipulations but the inner product it gives can be proved to have essentially the same value as our inner product.

\subsection{Cusp forms}

In this section, let $\Gamma$ be a congruence subgroup of $G_{\mathbb{Z}}$.

By linearity, we extend the definition $\left.f\right|_{k} \sigma$ to elements $\sigma$ of the group ring $\mathbb{R}[\Gamma]$. Let $k \geq 0$ be even and let $S_{k}(\Gamma)$ be the space of cusp forms of weight $k$.

We now define cusp forms of order $q$, which are the cuspidal analogues of the elements of $\tilde{M}_{k}^{q}$ discussed at the end of Sec. 2.1. First let $\tilde{S}_{k, 0}(\Gamma)=$ $S_{k, 0}(\Gamma)=S_{k}(\Gamma)$, so classical cusp forms are of order 0 . Next suppose $\tilde{S}_{k, q}(\Gamma)$ and $S_{k, q}(\Gamma)$ are already defined and let $\tilde{S}_{k, q+1}(\Gamma)$ be the space of all functions $f$ with

- $f: \mathbb{H} \rightarrow \mathbb{C}$ holomorphic,

- $\left.f\right|_{k}(\gamma-1) \in \tilde{S}_{k, q}(\Gamma)$ for every $\gamma \in \Gamma$,

- for every cusp $c,\left(\left.f\right|_{k} \sigma_{c}^{\Gamma}\right)(z)=O\left(e^{-\alpha y}\right)$ as $y \rightarrow \infty$ for some $\alpha>0$ ("rapid decay at the cusps").

Further, let $S_{k, q+1}(\Gamma)$ be the set of all $f \in \tilde{S}_{k, q+1}(\Gamma)$ with $\left.f\right|_{k}(\gamma-1)=0$ for every parabolic element $\gamma$ of $\Gamma$. Note that for $f \in S_{k, q+1}(\Gamma)$ and $\gamma \in \Gamma$ one has $\left.f\right|_{k}(\gamma-1) \in S_{k, q}(\Gamma)$.

Compare $\tilde{S}_{k, 1}$ with $P S_{k, 2}$ of [4] where a classification and a converse-theoremtype of result for such functions is proved.

Note that the space $V$ of all holomorphic functions on $\mathbb{H}$ which satisfy the above growth condition at each cusp, serves as a $G$-module, where, for instance, $G=\mathrm{SL}_{2}(\mathbb{Q})$ and $\Gamma=\mathrm{SL}_{2}(\mathbb{Z})$. In this way one obtains a theory of Hecke operators for the space $\tilde{S}_{k, q}(\Gamma) / \tilde{S}_{k, q-1}(\Gamma)$. Also, in view of the discussion at the end of Section 2.1., we have an inner product on the same space that makes it a unitary Hecke module. 


\subsection{Fourier-Taylor expansion}

Set $T=\left(\begin{array}{ll}1 & 1 \\ 0 & 1\end{array}\right)$ and let $V_{0}$ be the space of all holomorphic functions $f$ on the upper half plane with $f_{0} \mid T=f$, i.e. periodic with period 1. Inductively, let $V_{q+1}$ be the space of all holomorphic functions on $\mathbb{H}$ such that $\left.f\right|_{0}(T-1) \in$ $V^{q}$. We also set $V_{-1}=\{0\}$. Note that $S_{k, q}(\Gamma) \subset V_{q}$ if $\Gamma$ contains the translation $z \mapsto z+1$.

Proposition 2.3.1 Every $f \in V_{q}$ has a Fourier-Taylor-expansion

$$
f(z)=\sum_{n \in \mathbb{Z}} e^{2 \pi i n z}\left(a_{n, 0}+a_{n, 1} z+\cdots+a_{n, q} z^{q}\right)
$$

for uniquely determined coefficients $a_{k, j} \in \mathbb{C}$. For every $j$ and every $y>0$ the sequence $\left(a_{n, j} e^{-2 \pi n y}\right)_{n \in \mathbb{N}}$ is rapidly decreasing. The map $T: f \mapsto f(z+$ $1)-f(z)$ is a surjection from $V_{q}$ to $V_{q-1}$. With the natural inclusion $V_{0} \hookrightarrow V_{q}$ one gets an exact sequence

$$
0 \rightarrow V_{0} \rightarrow V_{q} \rightarrow V_{q-1} \rightarrow 0
$$

Proof: By induction on $q$. For $q=0$, every $f \in V_{0}$ is periodic, therefore has a Fourier-expansion, which, as $f$ is holomorphic, is of the form $f(z)=$ $\sum_{n} a_{n} e^{2 \pi i n z}$. Next assume the claim proven for $q$ and let $f \in V_{q+1}$. By induction,

$$
f(z+1)-f(z)=\sum_{n} e^{2 \pi i n z}\left(a_{n, 0}+\cdots+a_{n, q} z^{q}\right) .
$$

There is a unique sequence of numbers $b_{n, j}$ that satisfy the recursion relations

$$
a_{n, k}=\sum_{j=k+1}^{q+1}\left(\begin{array}{l}
j \\
k
\end{array}\right) b_{n, j}
$$

for $k=0, \ldots, q$. For each $y>0$ the sequence $b_{n, j} e^{-2 \pi n y}$ is rapidly decreasing. Let $g(z)=\sum_{n} e^{2 \pi i n z}\left(b_{n, 1} z+\cdots+b_{n, q+1} z^{q+1}\right)$, then $g$ is holomorphic, and

$$
g(z+1)-g(z)=\sum_{n} e^{2 \pi i n z}\left(a_{n, 0}+\cdots+a_{n, q} z^{q}\right)=f(z+1)-f(z) .
$$


So $h(z)=f(z)-g(z) \in V_{0}$. As $g$ and $h$ possess Fourier-Taylor expansions, so does $f$. By induction, the coefficients $a_{n, k}$ are uniquely determined, so are those of $h(z)$, which implies that the coefficients of $f$ are uniquely determined.

\subsection{Intervention of Lie groups}

For $\left(\begin{array}{cc}a & -b \\ b & a\end{array}\right) \in K_{\mathbb{R}}=\mathrm{SO}(2)$ define

$$
\varepsilon_{k}\left(\begin{array}{cc}
a & -b \\
b & a
\end{array}\right)=(a+i b)^{-k} .
$$

Let $G_{\mathbb{R}}=A N K_{\mathbb{R}}$ be the Iwasawa decomposition and let $\underline{k}: G_{\mathbb{R}} \rightarrow K_{\mathbb{R}}$ be the corresponding projection. Then

$$
\underline{k}\left(\begin{array}{ll}
a & b \\
c & d
\end{array}\right)=\frac{1}{\sqrt{c^{2}+d^{2}}}\left(\begin{array}{cc}
d & -c \\
c & d
\end{array}\right) .
$$

For a given function $f$ on the upper half plane define the function $\psi_{f}$ on $G_{\mathbb{R}}$ by

$$
\psi_{f}(g)=(\operatorname{Im}(g i))^{k / 2} \varepsilon_{k}(\underline{k}(g)) f(g i) .
$$

The next lemma shows that, via the identification $\psi$, the action $\left.\right|_{k}$ on functions on $\mathbb{H}$ becomes action by left translation.

Lemma 2.4.1 For $\gamma \in G_{\mathbb{R}}$ and $x \in G_{\mathbb{R}}$ one has

$$
\psi_{f}(\gamma x)=\psi_{\left.f\right|_{k} \gamma}(x) .
$$

Proof: A computation relying on the identity

$$
\varepsilon_{k}(\underline{k}(\gamma g))=\left(\frac{c z+d}{|c z+d|}\right)^{-k} \varepsilon_{k}(\underline{k}(g)),
$$

where $z=g i \in \mathbb{H}$. 
We next define a sequence of Hilbert spaces $L_{q}^{2}(\Gamma \backslash G)$, where $q=0,1,2, \ldots$. We start with providing spaces of measurable functions. Define $\mathcal{F}_{-1}(\Gamma \backslash G)=$ 0 and let $\mathcal{F}_{0}(\Gamma \backslash G)$ be the space of all $\Gamma$-invariant measurable functions on $G$. Inductively, define $\mathcal{F}_{q+1}$ to be the set of all measurable functions $f$ on $G$ with $(\gamma-1) f \in \mathcal{F}_{q}$ for every $\gamma \in \Gamma$. Let $\overline{\mathcal{F}}_{q+1}=\mathcal{F}_{q+1} / \mathcal{F}_{q}$. Consider the map $\eta: \mathcal{F}_{q+1} \rightarrow \operatorname{Hom}\left(\Gamma, \overline{\mathcal{F}}_{q}\right)$ given by

$$
\eta(f)(\gamma)=(\gamma-1) f
$$

This indeed defines a group homomorphism in $\gamma$, as for $\gamma, \tau \in \Gamma$ one has $(\gamma \tau-1) \equiv(\gamma-1)+(\tau-1)$ modulo $I^{2}$, and $I^{2} \mathcal{F}_{q+1} \subset \mathcal{F}_{q-1}$. The kernel of $\eta$ equals $\mathcal{F}_{q}$, so $\eta$ defines an injection $\overline{\mathcal{F}}_{q+1} \hookrightarrow \operatorname{Hom}\left(\Gamma, \overline{\mathcal{F}}_{q}\right) \cong \operatorname{Hom}(\Gamma, \mathbb{C}) \otimes \overline{\mathcal{F}}_{q}$. By iteration one gets an injective map

$$
\eta_{q}: \overline{\mathcal{F}}_{q} \hookrightarrow \operatorname{Hom}(\Gamma, \mathbb{C})^{\otimes q} \otimes \mathcal{F}_{0},
$$

where we have used $\overline{\mathcal{F}}_{0}=\mathcal{F}_{0}$.

Lemma 2.4.2 Suppose that $\Gamma$ is torsion-free. Then $\eta_{q}$ is surjective.

Proof: If the group $\Gamma$ has genus $g$ and $s$ cusps, then there are $2 g$ hyperbolic elements $\gamma_{i}$ and $s$ parabolic elements $\gamma_{2 g+i}$ generating $\Gamma$ and satisfying the relation:

$$
\left[\gamma_{1}, \gamma_{g+1}\right] \ldots\left[\gamma_{g}, \gamma_{2 g}\right] \gamma_{2 g+1} \cdots \gamma_{2 g+s}=1 .
$$

Because of this relation, every element in $\operatorname{Hom}(\Gamma, \mathbb{C})^{\otimes q} \otimes \mathcal{F}_{0}$ is uniquely determined by its values at $\left(\gamma_{i_{1}}, \ldots \gamma_{i_{q}}\right)$ for $i \in\{1, \ldots, N\}^{q}$, where $N=$ $\max (2 g, 2 g+s-1)$. Therefore, to establish surjectivity, it suffices to show that for every choice of functions $f_{i_{1}, \ldots, i_{q}} \in \mathcal{F}_{0}$ there is an $F \in \overline{\mathcal{F}}_{q}$ such that

$$
\left(\gamma_{i_{q}}-1\right) \ldots\left(\gamma_{i_{1}}-1\right) F=f_{i_{1}, \ldots, i_{q}} .
$$

By proposition 4.1 of [7], for every $q$-tuple of integers $L=\left(l_{1}, \ldots, l_{q}\right)$ in $\{1, \ldots, N\}$ there is a smooth function $\Lambda_{L}$ on $\mathbb{H}$ such that

$$
\left.\Lambda_{L}\right|_{0}\left(\gamma_{i_{1}}-1\right) \ldots\left(\gamma_{i_{q}}-1\right)=(-1)^{q} \delta_{I}^{L}
$$

for any $s$-tuple $I$, where $\delta_{I}^{L}$ is the Kronecker delta function of the $q$-tuple, namely $\prod_{k} \delta_{i_{k}}^{l_{k}}$. Then, with Lemma 2.4.1, the function

$$
F:=\sum_{L} f_{L} \psi_{\Lambda_{L}}
$$


with the sum ranging over all $L$ with components in $\{1, \ldots, N\}$ satisfies $(2)$.

Next, let $\mathcal{N}_{0}$ denote the space of all nullfunctions on $G$. Let

$$
\mathcal{N}_{q} \stackrel{\text { def }}{=} \eta_{q}^{-1}\left(\operatorname{Hom}(\Gamma, \mathbb{C})^{\otimes q} \otimes \mathcal{N}_{0}\right)
$$

Let $\mathcal{L}_{q}^{2}=\eta_{q}^{-1}\left(\operatorname{Hom}(\Gamma, \mathbb{C})^{\otimes q} \otimes \mathcal{L}^{2}(\Gamma \backslash G)\right)$, where $\mathcal{L}^{2}(\Gamma \backslash G)$ is the space of all square integrable functions on $\Gamma \backslash G$. Finally, we define

$$
L_{q}^{2}(\Gamma \backslash G) \stackrel{\text { def }}{=} \mathcal{L}_{q}^{2} / \mathcal{N}_{q}
$$

The space $L^{2}(\Gamma \backslash G)=\mathcal{L}^{2}(\Gamma \backslash G) / \mathcal{N}_{0}$ is a Hilbert space. Fix a Hilbert space structure on $\operatorname{Hom}(\Gamma, \mathbb{C})$ and equip the space $L_{q}^{2}(\Gamma \backslash G)$, which is mapped bijectively onto $\operatorname{Hom}(\Gamma, \mathbb{C})^{\otimes q} \otimes L^{2}(\Gamma \backslash G)$ the induced Hilbert space structure.

\subsection{No intervention of adèles}

Let $\mathbb{A}=\mathbb{A}_{\text {fin }} \times \mathbb{R}$ be the adele-ring over $\mathbb{Q}$. Let $K_{\Gamma}$ be a compact open subgroup of $G_{\mathbb{A}_{\text {fin }}}$, then $\Gamma=K_{\Gamma} \cap G_{\mathbb{Q}}$ is a congruence subgroup, and the natural map

$$
\Gamma \backslash G_{\mathbb{R}} \rightarrow G_{\mathbb{Q}} \backslash G_{\mathbb{A}} / K_{\Gamma},
$$

which maps $\Gamma x$ to $G_{\mathbb{Q}} x K_{\Gamma}$, is a $G_{\mathbb{R}^{-}}$equivariant, continuous bijection. This gives a natural isomorphism

$$
L^{2}(\Gamma \backslash G) \rightarrow L^{2}\left(G_{\mathbb{Q}} \backslash G_{\mathbb{A}}\right)^{K_{\Gamma}}
$$

In other words, automorphic forms on $\Gamma \backslash G$ can be lifted to $G_{\mathbb{Q}} \backslash G_{\mathbb{A}}$. This is what Dieudonné calls the "intervention of adeles". We ask for higher forms in the adelic setting. Note first that there are no higher $G_{\mathbb{Q}^{-}}$-invariants, as the group $G_{\mathbb{Q}}$ is perfect.

Also the $K_{\Gamma^{-a c t i o n}}$ does not yield higher order forms, at least not in the space $L^{2}\left(G_{\mathbb{Q}} \backslash G_{\mathbb{A}}\right)$, as the group $K_{\Gamma}$ is compact and acts through a continuous representation on the Hilbert space $L^{2}\left(G_{\mathbb{Q}} \backslash G_{\mathbb{A}}\right)$, see Proposition 1.1.1. 


\section{L-functions}

\subsection{Higher order cusp forms}

Let $\Gamma$ be a lattice in $G$ such that $\infty$ is a cusp of $\Gamma$ of width 1 . Let $f \in \tilde{S}_{k, q}(\Gamma)$. Then $f$ has the Fourier-Taylor expansion

$$
f(z)=\sum_{n=1}^{\infty} e^{2 \pi i n z}\left(a_{n, 0}+a_{n, 1} z+\cdots+a_{n, q} z^{q}\right) .
$$

For $\nu=0, \ldots, q$ define

$$
L_{\nu}(f, s)=\sum_{n=1}^{\infty} a_{n, \nu} n^{-s}
$$

Let

$$
\begin{aligned}
\Lambda(f, s) & =\int_{0}^{\infty} f(i y) y^{s-1} d y \\
& =\sum_{\nu=0}^{q} i^{\nu} \Gamma(s+\nu)(2 \pi)^{-(s+\nu)} L_{\nu}(f, s+\nu) .
\end{aligned}
$$

Let $S=\left(\begin{array}{cc}0 & -1 \\ 1 & 0\end{array}\right)$. After replacing $\Gamma$ with a conjugate if necessary, we may assume that 0 is a cusp of $\Gamma$, too. Then the group $S^{-1} \Gamma S$ has $\infty$ for a cusp. Let $w=w_{\Gamma}>0$ be its width. Let $S_{w}$ be the matrix $S \operatorname{diag}\left(\sqrt{w}, \sqrt{w}^{-1}\right)$. Then $\infty$ is a cusp of width 1 of the group

$$
\hat{\Gamma}=S_{w}^{-1} \Gamma S_{w} .
$$

Let $\hat{f}=\left.f\right|_{k} S_{w}$. Then $\hat{f} \in \tilde{S}_{k, q}(\hat{\Gamma})$. Note that $S_{w}^{2}=1$, and so $w_{\hat{\Gamma}}=w_{\Gamma}$ and $\hat{\hat{\Gamma}}=\Gamma$ as well as $\hat{\hat{f}}=f$.

As an example let $N \in \mathbb{N}$ and consider the group $\Gamma=\Gamma_{0}(N)$ consisting of all $\left(\begin{array}{ll}a & b \\ c & d\end{array}\right) \in \mathrm{SL}_{2}(\mathbb{Z})$ such that $c \equiv 0 \bmod N$. In this case one has $w=N$ and $\hat{\Gamma}=\Gamma$. 
Proposition 3.1.1 Assume that 0 and $\infty$ are cusps of $\Gamma$. For $f \in \tilde{S}_{k, q}(\Gamma)$ the function $\Lambda(f, s)$ extends to an entire function and satisfies the functional equation

$$
\Lambda(f, s)=i^{k} w^{s-\frac{k}{2}} \Lambda(\hat{f}, k-s) .
$$

For $0 \leq \nu \leq q-1$ the L-function $L_{\nu}(f, s)$ extends to an entire function.

Proof: Note that $\hat{f}(z)=(\sqrt{w} z)^{-k} f\left(\frac{-1}{w z}\right)$, so in particular one has $f\left(i \frac{1}{w y}\right)=$ $(i \sqrt{w} y)^{k} \hat{f}(i y)$. We decompose the integral defining $\Lambda(f, s)$ as $\int_{0}^{1 / \sqrt{w}}+\int_{1 / \sqrt{w}}^{\infty}$. The second gives an entire function $F(s)$. The first is

$$
\begin{aligned}
\int_{0}^{1 / \sqrt{w}} f(i y) y^{s} \frac{d y}{y} & =w^{-s} \int_{1 / \sqrt{w}}^{\infty} f\left(i \frac{1}{w y}\right) y^{-s} \frac{d y}{y} \\
& =i^{k} w^{-s+\frac{k}{2}} \int_{1 / \sqrt{w}}^{\infty} \hat{f}(i y) y^{k-s} \frac{d y}{y} \\
& =i^{k} w^{-s+\frac{k}{2}} \hat{F}(k-s),
\end{aligned}
$$

where $\hat{F}$ is the same as $F$ with $\hat{f}$ in place of $f$. Therefore, $\Lambda(f, s)=F(s)+$ $i^{k} w^{\frac{k}{2}-s} \hat{F}(k-s)$, which proves that $\Lambda(f, s)$ is entire and satisfies the functional quation.

Note that, with $\Lambda_{j}(f, s):=w^{s} \Gamma(s)(2 \pi)^{-s} L_{j}(f, s)$, we have that $\Lambda(f, s)$ equals $\sum_{\nu=0}^{q} i^{\nu} \Lambda_{\nu}(f, s+\nu)$, Form which the second claim follows in an easy induction.

\subsection{Convolution of L-functions}

In [1], it is shown that if $f(z)=\sum_{n=1}^{\infty} a_{n} e^{2 \pi i n z}$ and $g(z)=\sum_{n=1}^{\infty} b_{n} e^{2 \pi i n z}$ are cusp forms of weight $k \in \mathbb{Z}_{\geq 2}$ and 2 respectively, then the $n$-th Fourier coefficient of the second order form $F(z)=f(z) \int_{i \infty}^{z} g(w) d w$ is

$$
\sum_{j=1}^{n-1} \frac{a_{n-j} b_{j}}{j}
$$

Following [4], one can define the $L$-function of $F(z)$ by means of the Dirichlet series

$$
\sum_{n=1}^{\infty} n^{-s} \sum_{j=1}^{n-1} \frac{a_{n-j} b_{j}}{j}
$$


This function of $s$ admits meromorphic continuation and satisfies a functional equation ([4]).

Again we assume that 0 and $\infty$ are cusps of $\Gamma$, the width of $\infty$ being 1 . We define $w$, and $\hat{\Gamma}$ as in Theorem 3.1.1. Let $k, l$ be even integers $\geq 0$ and let $f \in S_{k}(\Gamma), g \in S_{l}(\Gamma)$. Denote their respective Fourier coefficients by $a_{n}$ and $b_{n}$ respectively. For complex numbers $s$ and $t$ with large enough real parts we set

$$
\left(L_{f} \# L_{g}\right)(s, t)=\sum_{n=1}^{\infty} n^{-s} \sum_{j=1}^{n-1} \frac{a_{n-j} b_{j}}{j^{t}} .
$$

For $\operatorname{Re}(s)$ large enough, we observe that the Mellin transform

$$
\Lambda_{f, g}(s, t)=\int_{0}^{\infty} f(i x) \int_{0}^{\infty} g(i x+i y) y^{t-1} d y x^{s-1} d x
$$

equals

$$
\begin{aligned}
& \sum_{n, m} a_{n} b_{m} \int_{0}^{\infty} \int_{0}^{\infty} e^{-2 \pi n x} e^{-2 \pi m x-2 \pi m y} y^{t-1} x^{s-1} d x d y= \\
& \left(\frac{1}{2 \pi}\right)^{s+t} \Gamma(s) \Gamma(t) \sum_{n, m} \frac{a_{n} b_{m}}{(n+m)^{s} m^{t}}=\left(\frac{1}{2 \pi}\right)^{s+t} \Gamma(s) \Gamma(t)\left(L_{f} \# L_{g}\right)(s, t)
\end{aligned}
$$

We shall analytically continue $\left(L_{f} \# L_{g}\right)(s, t)$ by a repeated application of the Riemann trick. We decompose the integrals into sums of the form $\int_{0}^{1 / \sqrt{w}}+\int_{1 / \sqrt{w}}^{\infty}$. Then $\Lambda_{f, g}(s, t)$ will be the sum of four terms,

$$
\underbrace{\int_{0}^{1 / \sqrt{w}} \int_{0}^{1 / \sqrt{w}}}_{=A}+\underbrace{\int_{0}^{1 / \sqrt{w}} \int_{1 / \sqrt{w}}^{\infty}}_{=B}+\underbrace{\int_{1 / \sqrt{w}}^{\infty} \int_{0}^{1 / \sqrt{w}}}_{=C}+\int_{1 / \sqrt{w}}^{\infty} \int_{1 / \sqrt{w}}^{\infty} .
$$

The last summand defines a holomorphic function on $\mathbb{C}^{2}$, as $f$ and $g$ are rapidly decreasing at $\infty$.

Recall that we have $f\left(i \frac{1}{w y}\right)=(i \sqrt{w} y)^{k} \hat{f}(y)$ and likewise for $g$ and $l$. The substitution $x \mapsto 1 / w x$ in the outer integral shows that $A$ equals

$$
\begin{aligned}
w^{-s} \int_{1 / \sqrt{w}}^{\infty} \int_{0}^{1 / \sqrt{w}} f\left(i \frac{1}{w x}\right) g\left(i\left(\frac{1}{w x}+y\right)\right) y^{t-1} x^{-s-1} d y d x= \\
i^{k} w^{-s+k / 2} \int_{1 / \sqrt{w}}^{\infty} \int_{0}^{1 / \sqrt{w}} \hat{f}(i x) g\left(i\left(\frac{1}{w x}+y\right)\right) y^{t-1} x^{k-s-1} d y d x
\end{aligned}
$$


As $f$ and $g$ are rapidly decreasing at $\infty$, this integral converges absolutely for $\operatorname{Re}(t)>0$ and defines a holomorphic function in $(s, t)$ in that region. The inner integral can be integrated by parts to get

$$
\left.\frac{1}{t} g\left(i\left(\frac{1}{w x}+y\right)\right) y^{t}\right|_{0} ^{1 / \sqrt{w}}-\frac{1}{t} \int_{0}^{1 / \sqrt{w}} y^{t} \frac{\partial}{\partial y} g\left(i\left(\frac{1}{w x}+y\right)\right) d y .
$$

So $A$ extends to a meromorphic function on $\operatorname{Re}(t)>-1$ with a simple pole at $t=0$. Iteration of that argument shows that $A$ is entire except for simple poles at $t=0,-1,-2, \ldots$.

The other summands can be treated similarly. Since the $\Gamma$-function has poles at $t=0,-1,-2, \ldots$, we have proven the following theorem.

Theorem 3.2.1 The function $L_{f} \# L_{g}$ extends to an entire function on $\mathbb{C}^{2}$.

If $t=p \in \mathbb{N}$ we set $\left(L_{f} \#_{p} L_{g}\right)(s)=\left(L_{f} \# L_{g}\right)(s, p)$. Then $\left(L_{f} \#_{p} L_{g}\right)(s)$ extends to an entire function for every $p \in \mathbb{N}$. Next we fix $p=l-1$, where $l$ is the weight of $g$. Then we can obtain a functional equation for a related Dirichlet series, which though involves "lower-order" $L$-functions $L_{f}, L_{g}$.

Specifically we consider

$$
\Lambda_{f, g}(s)=\int_{0}^{\infty} f(i x)\left(\int_{x}^{\infty}+\int_{x}^{0} g(i y)(y-x)^{l-2} d y\right) x^{s-1} d x
$$

The relation with values of $L_{f} \#_{l-1} L_{g}$ is given by

Proposition 3.2.2 If $L_{f}(s), L_{g}(s)$ are the L-functions of $f \in S_{k}, g \in S_{l}$, then

$$
\begin{aligned}
\Lambda_{f, g}(s)=-2\left(\frac{1}{2 \pi}\right)^{s+l-1}(l-2) ! \Gamma(s)\left(L_{f} \#_{l-1} L_{g}\right)(s)- \\
(l-2) !\left(\frac{1}{2 \pi}\right)^{s+l-1} \Gamma(s+j) \sum_{j=0}^{l-2} \frac{(-1)^{j}}{j !} L_{f}(s+j) L_{g}(l-j-1) .
\end{aligned}
$$

\section{Proof:}

$$
\begin{aligned}
& \Lambda_{f, g}(s)=2 \int_{0}^{\infty} f(i x)\left(\int_{x}^{\infty} g(i y)(y-x)^{l-2} d y\right) x^{s-1} d x- \\
& \quad \int_{0}^{\infty} f(i x)\left(\int_{0}^{\infty} g(i y)(y-x)^{l-2} d y\right) x^{s-1} d x
\end{aligned}
$$


From the binomial expansion of $(y-x)^{l-2}$ we deduce that this equals

$$
\begin{aligned}
& -2\left(\frac{i}{2 \pi}\right)^{s+l-1}(l-2) ! \Gamma(s)\left(L_{f} \# L_{g}\right)(s, l-1) \\
& \quad-\sum_{j=0}^{l-2}\left(\begin{array}{c}
l-2 \\
j
\end{array}\right)(-1)^{j}\left(\int_{0}^{\infty} f(i x) x^{s+j-1} d x\right)\left(\int_{0}^{\infty} g(i y) y^{l-2-j} d y\right)
\end{aligned}
$$

This gives the result.

The analytic continuation of $\Lambda_{f, g}$ can be deduced from this proposition together with Theorem (3.2.1) and the analytic continuation of $\Gamma(s) L_{f}(s)$. The functional equation is given by

Proposition 3.2.3 The function $\Lambda_{f, g}(s)$ satisfies

$$
\Lambda_{f, g}(s)=w^{(k-l) / 2-s+1} i^{s+k+1} \Lambda_{\hat{f}, \hat{g}}(k-l-s+2) .
$$

Proof: The change of variables $x \rightarrow 1 / w x$ implies that $\Lambda_{f, g}(s)$ equals

$$
w^{k / 2-s} \int_{0}^{\infty} \hat{f}(i x)\left(\int_{1 / w x}^{\infty}+\int_{1 / w x}^{0} g(i y)\left(y-\frac{1}{w x}\right)^{l-2} d y\right) x^{k-s-1} d x
$$

The same change of variables on $y$ gives

$$
-i^{-l} w^{(k-l) / 2-s+1} \int_{0}^{\infty} \hat{f}(i x)\left(\int_{x}^{\infty}+\int_{x}^{0} \hat{g}(i y)(x-y)^{l-2} d y\right) x^{k-s-l+1} d x
$$

which is the desired statement.

\section{References}

[1] Chinta, G.; Diamantis, N.; O'Sullivan, C.: Second order modular forms. Acta Arith. 103 (2002), no. 3, 209-223

[2] Conrey, J. B.; Farmer, D. W.: An extension of Hecke's converse theorem. Internat. Math. Res. Notices 1995, no. 9, 445-463.

[3] Deitmar, A.: Higher order group cohomology and the Eichler-Shimura map. to appear in: J. reine u. angew. Math. 
[4] Diamantis, N.; Knopp, M.; Mason, G.; O'Sullivan, C.: L-functions of second-order cusp forms. Ramanujan J. 12 (2006), no. 3, 327-347.

[5] Diamantis, N.; O'Sullivan, C.: The dimensions of spaces of holomorphic second-order automorphic forms and their cohomology. to appear in: Transactions of the AMS.

[6] Diamantis, N.; Sim, D.: The classification of higher-order cusp forms. to appear in: J. Reine Angew. Math.

[7] Diamantis, N.; Sreekantan, R.: Iterated integrals and higher order automorphic forms. Commentarii Mathematici Helvetici 81 (2006), 481494.

[8] Farmer, D.: Converse theorems and second order modular forms. AMS Sectional Meeting. Salt Lake City (2002).

[9] Goldfeld, D.: Modular forms, elliptic curves and the ABC-conjecture. A panorama of number theory or the view from Baker's garden (Z?rich, 1999), 128-147, UPs, Cambridge, 2002.

[10] Imamoglu, Ö.; Martin, Y.: A converse theorem for second-order modular forms of level $N$. Acta Arith. 123 (2006), no. 4, 361-376.

[11] Imamoglu, Ö.; O'Sullivan, C.: Higher-order cusp forms: inner products in preparation.

[12] Kleban, P.; Zagier, D.: Crossing probabilities and modular forms. J. Statist. Phys. 113 (2003), no. 3-4, 431-454

Mathematisches Institut, Auf der Morgenstelle 10, 72076 Tübingen, Germany, deitmar@uni-tuebingen.de

School of Mathematical Sciences, University of Nottingham, University Park, Nottingham NG7 2RD, United Kingdom

nikolaos.diamantis@maths.nottingham.ac.uk 